\title{
Intracellular Quantification and Localization of Label-Free Iron Oxide Nanoparticles by Holotomographic Microscopy
}

This article was published in the following Dove Press journal:

Nanotechnology, Science and Applications

\author{
Ralf P Friedrich $\mathbb{D}^{\prime}$ \\ Eveline Schreiber' \\ Rainer Tietze' \\ Hai Yang ${ }^{2}$ \\ Christian Pilarsky $\mathbb{( D}^{2}$ \\ Christoph Alexiou' \\ 'Department of Otorhinolaryngology, \\ Head and Neck Surgery, Section of \\ Experimental Oncology and \\ Nanomedicine (SEON), Else Kröner- \\ Fresenius-Stiftung-Professorship, \\ Universitätsklinikum Erlangen, Erlangen, \\ 91054, Germany; ${ }^{2}$ Department of \\ Surgery, Universitätsklinikum Erlangen, \\ Erlangen 91054, Germany
}

Background: The limitations of optical microscopy to determine the cellular localization of label-free nanoparticles prevent a solid prediction of the cellular effect of particles intended for medical applications. To avoid the strong physicochemical changes associated with fluorescent labelling, which often result in differences in cellular uptake, efficiency and toxicity of particles, novel detection techniques are required.

Methods: In the present study, we determined the intracellular content of unlabeled SPIONs by analyzing refractive index (RI)-based images from holotomographic three-dimensional (3D) microscopy and side scatter data measured by flow cytometry. The results were compared with the actual cellular SPION amount as quantified by atomic emission spectroscopy (AES).

Results: Live cell imaging by 3D holotomographic microscopy demonstrated cell-specific differences in intracellular nanoparticle uptake in different pancreatic cell lines. Thus, treatment of PANC-1 ${ }^{\text {SMAD4 (1-4) }}$ and PANC-1 ${ }^{\text {SMAD4 (2-6) }}$ with SPIONs resulted in a significant increase in number of areas with higher RI, whereas in PANC-1, SUIT-2 and PaCa DD183, only a minimal increase of spots with high RI was observed. The increase in areas with high RI was in accordance with the SPION content determined by quantitative iron measurements using AES. In contrast, determination of the SPION amount by flow cytometry was strongly cell typedependent and did not allow the discrimination between intracellular and membrane-bound SPIONs. However, flow cytometry is a very rapid and reliable method to assess the cellular toxicity and allows an estimation of the cell-associated SPION content.

Conclusion: Holotomographic 3D microscopy is a useful method to distinguish between intracellular and membrane-associated particles. Thus, it provides a valuable tool for scientists to evaluate the cellular localization and the particle load, which facilitates prediction of potential toxicity and efficiency of nanoparticles for medical applications.

Keywords: superparamagnetic iron oxide nanoparticles, cellular SPION uptake, cytotoxicity, flow cytometry, atomic emission spectroscopy

\section{Introduction}

Superparamagnetic iron oxide nanoparticles (SPIONs) play an increasingly important role in the development of improved contrast agents for diagnostic purposes. Moreover, their magnetic properties allow the use of directed application techniques such as magnetic drug targeting (MDT) or hyperthermia for the treatment of different diseases. Additionally, the specific surface functionalization can further adapt their biochemical properties and improve targeting in order to treat or
Correspondence: Ralf P Friedrich Department of Otorhinolaryngology, Experimental Oncology and Nanomedicine (SEON), Else KrönerFresenius-Stiftung-Professorship, Universitätsklinikum Erlangen, Glückstrasse 10a, Erlangen 91054, Germany

Tel +49 913। 8543943

Fax +4991318534828

Email ralf.friedrich@uk-erlangen.de
Nanotechnology, Science and Applications 2020:13 ||9-130 
visualize certain disease processes. ${ }^{1-4}$ The successful use of SPIONs depends both on the selected application method and on the specific properties of the particles. While particles used for blood-pool imaging should preferably not interact with cellular structures, SPIONs intended for MDT or hyperthermia should accumulate in the target area, or be integrated into certain cells or tissues. Especially in the field of cancer therapy and regenerative medicine, a direct interaction of particles with cells is necessary to achieve a maximum effect. In cancer treatment, the particles must be directed to the tumor site in order to exert their anti-carcinogenic activity, whereas in regenerative medicine, particle-loaded cells or spheroids are directed directly to the target region. The development, investigation and validation of such particle systems therefore require reliable techniques not only to quantify the cellular particle load but also to discriminate between cell membrane-associated and intracellular particles to ensure an efficient application.

At present, several methods of SPION quantification are in use, such as magnetic particle spectroscopy (MPS), ultraviolet spectrophotometry (UVS), atomic adsorption and emission spectroscopy (AAS, AES), as well as proton nuclear magnetic resonance spectroscopy ((1)H-NMR). ${ }^{5-11}$ However, none of these methods allows the discrimination between the particles localized inside the cell and particles attached to the cell membrane. Only a few techniques, such as transmission electron microscopy (TEM), cryogenic TEM (cryo-TEM), and confocal fluorescence microscopy, are capable to visualize the accumulation of particles within cells and their interactions with other cellular structures, enabling the investigation of internalization, trafficking and degradation or removal of nanostructures. ${ }^{12-14}$ While the resolution achievable by transmission electron microscopy techniques is still unconquerable, this method is only applicable if the underlying scientific question can be answered by investigation of non-living and fixed cells. In contrast, fluorescencebased microscopy, including super-resolution techniques such as LSM (laser scanning microscopy), SIM (Structured Illumination Microscopy), photoactivated localization microscopy (PALM) and stochastic optical resolution microscopy (STORM), allows the 3D examination of structures within living cells. However, the main disadvantage of these techniques is the imperative need of fluorophore labelling, which affects the physicochemical properties of the particles and their binding kinetics to cells, and may therefore seriously alter the results of cell-based experiments. ${ }^{15}$ Additionally, most of these methods are unsuitable for a rapid evaluation of 3D localization of label-free particles within living cells. Meanwhile, several techniques have been developed to allow a label-free, site-specific detection of nanoparticles, such as surface plasmon resonance imaging (SPRI), a technique that can image refractive index changes at an interface, or hyperspectral dark-field and reflected light microscopy (HDM, HRLM), which measures the spectrum for each pixel. ${ }^{16-19}$ Another promising method that allows the 3D imaging of unstained living cells and cellular structures is called holotomographic microscopy and is based on the changes in refractive index. ${ }^{20}$ In this study, we investigated the feasibility of the holotomographic technique for estimating the cellular SPION uptake in different cell lines. As a model system, we used different pancreatic cell lines and incubated them with lauric acid/albumin hybrid coated SPIONs (SPION ${ }^{\mathrm{LA}-\mathrm{BSA}}$ ). ${ }^{21}$ The results were compared to and validated by fluorescence microscopy, side scatter analysis by flow cytometry, and by quantitative iron analysis using microwave plasma-atomic emission spectrometry (MPAES). ${ }^{10,22}$

We report here that the holotomographic microscopy allows the determination of SPION localization inside the living cells. A good correlation was found between cellular SPION amounts as estimated by holotomographic imaging and the iron content by AES. Moreover, a strong cell line dependency of the side scatter data and SPION toxicity measured by multicolor flow cytometry was observed.

\section{Materials and Methods \\ Materials}

Iron (II) chloride tetrahydrate $\left(\mathrm{FeCl}_{2} \bullet 4 \mathrm{H}_{2} \mathrm{O}\right)$, iron (III) chloride hexahydrate $\left(\mathrm{FeCl}_{3} \bullet 6 \mathrm{H}_{2} \mathrm{O}\right)$, Eppendorf ultrafiltration tubes with a molecular weight cut-off (MWCO) of 3 $\mathrm{kDa}$ and bovine serum albumin were purchased from Merck (Darmstadt, Germany). Iron reference standards were bought from Bernd Kraft $\mathrm{GmbH}$ (Duisburg, Germany). Sterile Rotilabo ${ }^{\circledR}$ syringe filters with cellulose mixed ester membranes were supplied by Roth (Karlsruhe, Germany). Propidium iodide (PI), sodium citrate, triton X-100 were purchased from Sigma-Aldrich (St Louis, MO, USA). Ringer's solution was obtained from Baxter Healthcare (Zürich, Switzerland). Fetal bovine serum (FBS) superior, were purchased from Thermo Fisher Scientific (Waltham, MA, USA). DMEM was obtained from Th Geyer (Renningen, Germany), EMEM from ATCC (Manassas, VA, USA), RPMI 1640 from Life Technologies (Carlsbad, CA, USA), Keratinocyte SFM 
from Gibco (Dublin, Ireland). Water used in all experiments was of ultrapure quality produced by the Milli-Q ${ }^{\circledR}$ system from Merck (Darmstadt, Germany).

\section{Nanoparticle Production}

Lauric acid/albumin hybrid-coated SPIONs (SPION ${ }^{\text {LA-BSA }}$ ) were synthetized as previously described. ${ }^{21}$ In brief, SPION $^{\mathrm{LA}}$ were produced by co-precipitation of iron (III) chloride and iron (II) chloride under argon atmosphere in alkaline media and subsequent in situ coating with lauric acid. SPION ${ }^{\text {LA-BSA }}$ were produced by mixing of SPION ${ }^{\mathrm{LA}}$ particles with a freshly prepared $20 \%$ bovine serum albumin solution followed by incubation for 30 minutes under constant stirring. After purification and concentration by KrosFlo (molecular weight cut-off $100 \mathrm{kDa}$; KrosFlo ${ }^{\circledR}$ Research IIi Tangential Flow Filtration System, Spectrum Laboratories, Rancho Dominguez, California, USA), particles were sterilized by filtration through a $0.22 \mu \mathrm{m}$ membrane filter.

\section{Quantification of SPION Stock Concentrations by MP-AES}

The SPION solution was diluted with ultrapure water and dissolved in $65 \%$ nitric acid for 10 minutes at $95^{\circ} \mathrm{C}$. After cooling to room temperature, samples were further diluted with $\mathrm{H}_{2} \mathrm{O}$. The iron content was then determined by an Agilent 4200 microwave plasma-atomic emission spectrometer (MP-AES) (Agilent Technologies, Santa Clara, CA, USA) using a commercial iron solution ranging from 0.05 to $5.0 \mathrm{mg} / \mathrm{L}$ as an external standard.

\section{Dynamic Light Scattering (DLS) and Zeta Potential Measurements}

The hydrodynamic size and Zeta potential in water were determined using a Malvern Zetasizer Nano ZS (Malvern Instruments, Worcestershire, England). The nanoparticles were diluted with ultrapure water to a total iron concentration of $30 \mu \mathrm{g} / \mathrm{mL}$ and measured in triplicates at $25^{\circ} \mathrm{C}$.

\section{Cell Line Production}

PaCa DD183, PANC-1 ${ }^{\text {SMAD4 (1-4) }}$ and PANC-1 SMAD4 (2-6) were produced in the Department of Surgery, Universitätsklinikum Erlangen, Germany. The PANC$1^{\text {SMAD4 }}$ knockout cell lines were generated by using the CRISPR-Cas9 system on PANC-1 cells based on the protocol provided by Ran et al. ${ }^{23}$ NC-human-sgRNA-forward (5'-caccgATCGTATCATCAGCTAGCGC-3'), NC-humansgRNA-reverse (5'-aaacGCGCTAGCTGATGATACGATc
-3'), SMAD4-human-sgRNA1-forward (5'-caccgAAC TCTGTACAAAGACCGCG-3'), SMAD4-human-sgRNA1 -reverse (5'-aaacCGCGGTCTTTGTACAGAGTTc-3'), SMAD4-human-sgRNA2-forward (5'-caccgAGTCC TACTTCCAGTCCAGG-3'), SMAD4-human-sgRNA2reverse (5'-aaacAGTCCTACTTCCAGTCCAGGc-3') were synthesized by Eurofins (Luxembourg). sgRNAs were cloned into plasmid pSpCas9(BB)-2A-GFP (PX458, addgene Plasmid No. 48138, Watertown, MA, USA) and confirmed by sequencing. PANC-1 cells were transfected with negative control plasmid and SMAD4 knockout plasmids using Lipofectamine 3000 Transfection Reagent (Invitrogen, Catalog No. L3000015, Waltham, MA, USA). A fluorescence-activated cell sorter sorted all GFP (Green fluorescent protein) positive cells. Forty-eight GFP positive cells were seeded into a 96-well plate for single clone's selection. Western blot was performed to detect the knockout effect using SMAD4 antibody (Santa Cruz Biotechnology, Inc. sc-7966). The PaCa DD183 cell line was derived from a pancreatic ductal adenocarcinoma originated from peritoneal metastases by the Dresden outgrowth protocol as described in Ruckert et al. ${ }^{24}$ Briefly, the tumor tissue was minced and adhered to a 6-well plate. The tumor tissue pieces were then incubated for 14 days for the outgrow of epithelial cells. The cell line was characterized by examination of their morphology and their cytostructural and functional profiles. The generation of the cell lines from human pancreatic ductal adenocarcinomas was approved by the local ethics committee at the University Hospital Erlangen (EK304122007).

\section{Cell Culture}

PANC-1, a human pancreatic carcinoma of ductal origin were purchased from ATCC (Manassas, VA, USA) and SUIT-2, a human pancreatic cancer cell line established from liver metastasis from JCRB Cell Bank (Osaka, Japan). PANC-1 were cultivated in DMEM with 9\% fetal bovine serum and 0.9\% L-Glutamin, SUIT-2 in EMEM and 9.1\% FBS, PaCa DD183 in DMEM, 13\% FBS and 22\% Keratinocyte-SFM,

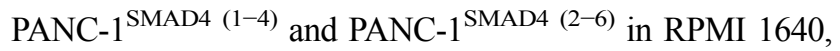
$9 \%$ FCS and $0.9 \%$ L-Glutamine. All cell lines were cultivated at $37^{\circ} \mathrm{C}$ and $5.0 \% \mathrm{CO}_{2}$. For further passaging, trypsinization was performed using Trypsin-EDTA according to the manufacturer's instructions.

\section{Holotomographic Imaging}

For experiments, 5000 cells were seeded into $32 \mathrm{~mm} \mu$ Dishes (Ibidi, Gräfelfing, Germany). After 24 h, aqueous 
SPION $^{\text {LA-BSA }}$ were added to a final concentration of 100 $\mu \mathrm{g}_{\mathrm{Fe}} / \mathrm{mL}$ cell culture media, whereas control samples were supplemented with the same amount of ultrapure water. Cells were incubated for another $24 \mathrm{~h}$, washed twice with PBS and imaged under controlled temperature and atmosphere conditions at $5 \% \mathrm{CO}_{2}$ and $37^{\circ} \mathrm{C}$ by live cell $3 \mathrm{D}$ holotomographic microscopy (Fluo-3D Cell Explorer ${ }^{\circledR}$, Nanolive, Tolochenaz, Switzerland). Digital staining and $3 \mathrm{D}$ rendering of images and data export were performed by Steve software v.1.6.3496 ${ }^{\circledR}$ (Nanolive, Tolochenaz, Switzerland). In short, the refractive index is measured in three dimensions using a low power class I laser $(0.2 \mathrm{~mW} /$ $\mathrm{mm}^{2}, \lambda 520 \mathrm{~nm}$ ) and a 60x dry objective with a Numerical Aperture of 0.8 . Depending on the intensity, the refractive index can be digitally stained to visualize cells, cell components and clusters of nanoparticles. In 2D view, every single frame can be viewed and selected. In 3D view, the sample can be rotated in all directions and cut at every position to closely investigate the localization of cellular components. For quantification, the settings of the digitally staining can be saved and transferred to other images to ensure the comparability of the stained volume, which is displayed in the software's panel viewer. To achieve a strong significance, each sample were investigated in three experiments containing at least 26 images with a total of 200-400 single cells. Finally, the RI volume of each image was normalized to the cell number and data sets were analyzed using Microsoft Excel.

\section{Cellular SPION Uptake and Toxicity by Flow Cytometry}

Cell granularity and cell viability were determined by flow cytometry using a Gallios cytofluorometer (Beckman Coulter, Fullerton, CA, USA). ${ }^{22,25}$ Cells were seeded into 6-well cell culture plates (TPP, Techno Plastic Products AG, Trasadingen, Switzerland) in a total volume of $2.5 \mathrm{~mL}$. The amount of seeded cells depended on the growth rate of the individual pancreatic cell lines and was calculated to achieve a final confluency of $95 \%$ after $48 \mathrm{~h}$. After $24 \mathrm{~h}, \mathrm{SPION}^{\mathrm{LA}-}$ BSA was added to a final concentration of $100 \mu \mathrm{g}_{\mathrm{Fe}} / \mathrm{mL}$ cell culture media, which corresponds to $28.0 \mu \mathrm{g}_{\mathrm{Fe}} / \mathrm{cm}^{2}$ cell culture plate area. The negative control contained $0 \mu \mathrm{g}_{\mathrm{Fe}} / \mathrm{mL}$ cell culture media, and the toxicity control 2.0\% DMSO. Subsequently, cells were incubated for another $24 \mathrm{~h}$ before analysis. The 6-well samples were harvested by trypsination and centrifugation before the cell pellets were resuspended in $0.5 \mathrm{~mL}$ PBS. Cell suspensions were used to determine the absolute cell counts with the MUSE ${ }^{\circledR}$ Cell Analyzer (MerckMillipore, Billerica, MA, USA), as well as for flow cytometry analysis and SPION quantification measurements using MP-AES. For cell death analysis, $50 \mu \mathrm{L}$ of the cell suspensions were incubated with $250 \mu \mathrm{L}$ of freshly prepared staining solution containing $1 \mu \mathrm{g} / \mathrm{mL}$ annexin V-FITC, $1 \mu \mathrm{g} / \mathrm{mL}$ Hoechst 33342, and $5.1 \mu \mathrm{g} / \mathrm{mL} \quad 1,1^{\prime}, 3,3,3^{\prime}, 3^{\prime}-$ hexamethylindodicarbocyanine iodide (DiIC1(5)) (all from Life Technologies, Darmstadt, Germany), $20 \mu \mathrm{g} / \mathrm{mL}$ propidium iodide (Sigma-Aldrich, Taufkirchen, Germany) in Ringer's solution (Fresenius Kabi AG, Bad Homburg, Germany) for 20 minutes at $4{ }^{\circ} \mathrm{C} .^{25}$ Electronic compensation was used to eliminate bleed through fluorescence. Data analysis was performed with Kaluza software version 2.0 (Beckman Coulter, Fullerton, CA, USA). A visual representation of the gating strategies are shown in supplementary Figure S1. All flow cytometry analyses were conducted in four independent experiments with quadruplicates.

\section{Quantification of Cellular SPIONs by MP-AES}

Cell suspensions were used to determine the absolute cell counts and viability with the MUSE ${ }^{\circledR}$ Cell Analyzer (MerckMillipore, Billerica, USA). A total of $200 \mu \mathrm{L}$ cell suspension was centrifuged for $5 \mathrm{~min}$ at $1,000 \times \mathrm{g}$. The supernatants were aspirated and dissolved in $50 \mu \mathrm{L}$ nitric acid $65 \%$, incubated at $90^{\circ} \mathrm{C}$ for $15 \mathrm{~min}$ with a shaking incubator and diluted to $450 \mu \mathrm{L}$ with ultrapure water. The total iron content of the cells was then determined by MP-AES. The calibration curve was prepared with iron concentrations ranging from $0.05 \mathrm{mg} / \mathrm{L}$ to $5.0 \mathrm{mg} / \mathrm{L}$. The iron content was normalized to the total number of cells in the analyzed cell pellet.

\section{Imaging of SPION-Treated Cells After Fluorescent Staining}

Twenty-five thousand cells were seeded on gelatin-coated coverslips. After $24 \mathrm{~h}$, cells were treated with SPIONs (100 $\mu \mathrm{g}_{\mathrm{Fe}} / \mathrm{mL}$ ) and incubated for another $24 \mathrm{~h}$ before imaging of cellular F-actin, SPION aggregates and nuclei. After washing, the cells were fixed for 20 min with $4 \%$ PBS buffered formalin and permeabilized for $3 \mathrm{~min}$ with Triton X-100 (0.5\% in PBS). After thoroughly washing with PBS, cells were incubated for 20 min with Alexa Fluor 488 Phalloidin (1:100) to visualize F-actin and Hoechst 33334 (1:2500) (Molecular Probes, Life Technologies, Carlsbad, CA, USA) to visualize nuclei, followed by washing 3 times with PBS. Finally, stained cells were imaged with a Zeiss Axio 
Observer. Z1 fluorescent microscope (Carl Zeiss AG, Oberkochen, Germany).

\section{Results SPION Characterization}

In this study, lauric acid/albumin hybrid coated SPIONs (SPION $^{\mathrm{LA}-\mathrm{BSA}}$ ) were synthesized according to the previously described protocol by co-precipitation of iron (III) chloride and iron (II) chloride under argon atmosphere in alkaline media and subsequent in situ coating with lauric acid. ${ }^{21}$ The resulting SPION ${ }^{\mathrm{LA}}$ particles were mixed with a freshly prepared $20 \%$ bovine serum albumin solution and purified by centrifugal ultrafiltration. ${ }^{26}$ In water, the hydrodynamic diameter of SPION ${ }^{\text {LA-BSA }}$ was $88.1 \pm 3.1 \mathrm{~nm}$ and the zeta potential revealed a negative value of $-34.1 \mathrm{mV} \pm 0.2$. These SPIONs are known to be well tolerated by the relatively sensitive primary human umbilical vein endothelial cells (HUVECs) and are readily incorporated into the cytoplasm. ${ }^{22}$ We therefore assumed that those particles are also appropriate for uptake experiments with other cell lines, such as pancreatic cells. To verify this and to ensure the suitability of these cells for the experiments with SPIONs, we first determined the nanoparticle cytotoxicity by flow cytometry, which allows the analysis of multiple toxicity parameters. ${ }^{27-29}$ Notably, all investigated cell lines adhered extremely strongly to the surface of the cell culture dishes, resulting in a necessity of relatively harsh sample preparation, which caused an overall slight reduction in cell viability. However, as shown by Annexin $\mathrm{V}$ and propidium iodide staining, SPION treatment did not result in a significant change in the numbers of either viable, necrotic or apoptotic cells, except for SUIT-2 cells, where the viability dropped from $76.3 \%$ to $62.3 \%$ upon incubation with SPIONs (Figure 1). In the same cell type, incubation with $2 \%$ DMSO as a toxicity control lead to more pronounced effects (viability reduction by $34.6 \%$ ), indicating a diverse cellular sensitivity towards different substances. Interestingly, some of the tested cell types were resistant to DMSO treatment: While the viability compared to the control sample were reduced by $14.3 \%$ in PaCa DD183 and to $19.3 \%$ in PANC1 cells there were no detectable effects of DMSO on PANC-1 ${ }^{\text {SMAD4 (1-4) }}$, and PANC-1 ${ }^{\text {SMAD4 (2-6) even showed }}$ a higher viability after the incubation with DMSO. Similar results were achieved when analyzing the status of the mitochondrial membrane potential by DiIC1(5) staining (Figure S2) ${ }^{25}$ SPION $^{\text {LA-BSA }}$ had only a slight negative

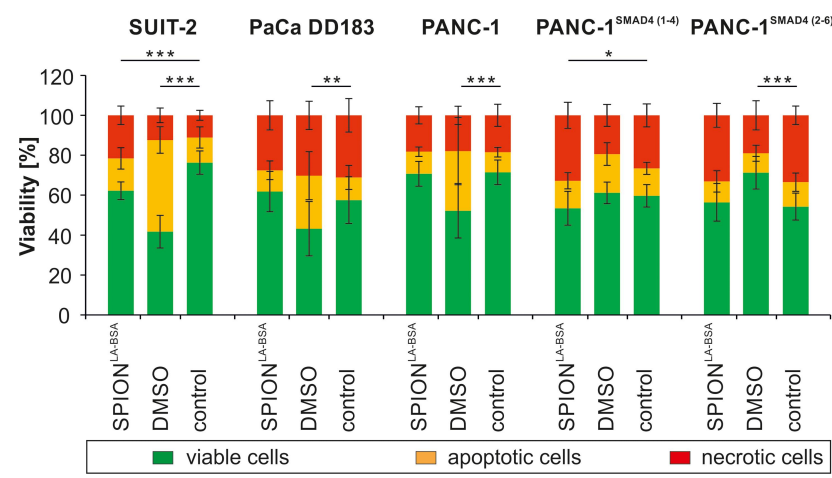

Figure I Viability of different pancreatic cell lines after SPION treatment. Cells were incubated for $24 \mathrm{~h}$ with $100 \mu \mathrm{g}_{\mathrm{Fe}} / \mathrm{mL}$ SPION ${ }^{\mathrm{LA}-\mathrm{BSA}}$. Cell viability was determined by Annexin V-FITC/propidium iodide (AxV/PI) staining and analyzed by flow cytometry. The amount of viable (AxV-PI-), apoptotic ( $\mathrm{AxV}+\mathrm{PI}-)$ and necrotic $(\mathrm{PI}+)$

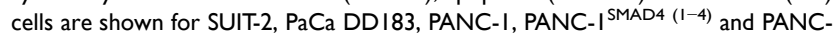
$I^{\text {SMAD4 (2-6) }}$. Toxicity controls contain $2 \%$ DMSO, negative controls represent the corresponding amount of $\mathrm{H}_{2} \mathrm{O}$ instead of water-based ferrofluid. Data are expressed as the mean \pm standard deviation ( $n=4$ with technical quadruplicates). Statistical significance in the percentage of viable cells are indicated with $*, * *$ and ***. The respective confidential intervals are $p<0.01, p<0.005$ and $p<0.00002$ and were calculated via $t$-test analysis.

Abbreviations: SPION, superparamagnetic iron oxide nanoparticles; SPION ${ }^{\text {LA-BSA }}$, lauric acid- and bovine serum albumin-coated SPIONs; DMSO, dimethyl sulfoxide.

impact on SUIT-2 cells, whereas the other cell lines were not significantly affected by the treatment with particles.

\section{Determination of Cellular SPIONs by Holotomographic Microscopy}

The cellular and cell membrane-associated SPION content were previously shown to be dependent on the SPION concentration added to the cell culture medium. ${ }^{10,30}$ However, the applied methods did not discriminate between incorporated SPIONs and SPIONs bound to the cell membrane. Here, we used a non-invasive and labelfree holotomographic live cell imaging microscope based on the specific refractive index (RI) of cells and cell components to determine the localization of SPIONs. The results were subsequently compared with other methods such as microscopy, atomic emission spectroscopy and flow cytometry. For the holotomographic imaging, we incubated different pancreatic cell lines with a defined concentration of SPION ${ }^{\text {LA-BSA }}\left(100 \mu \mathrm{g}_{\mathrm{Fe}} / \mathrm{mL}\right)$ and determined the cellular localization and amount of the accumulated particles after $24 \mathrm{~h}$. Figure $2 \mathrm{~A}-\mathrm{E}$ show representative refractive index-based $2 \mathrm{D}$ and $3 \mathrm{D}$ images of SPIONtreated cells. Digitally stained 3D images, in which different cellular structures as well as nanoparticles can be quantified, are illustrated in Figure 2C-E.

To expose the full range of variation in cellular SPION uptake, the areas of high RI, representing accumulated 
A

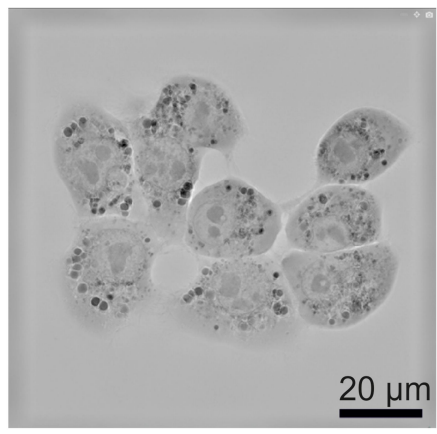

C

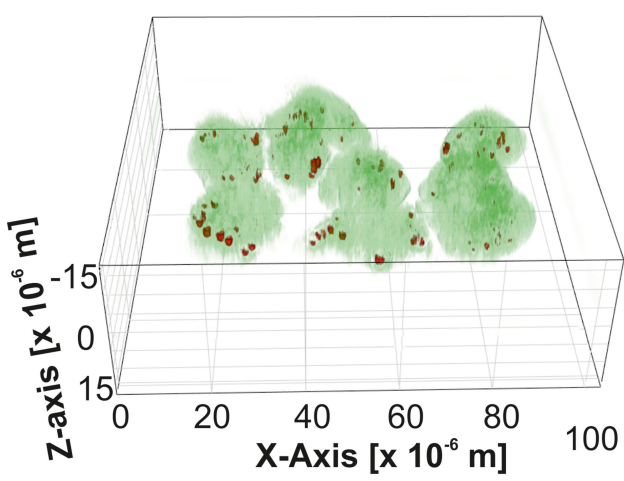

B

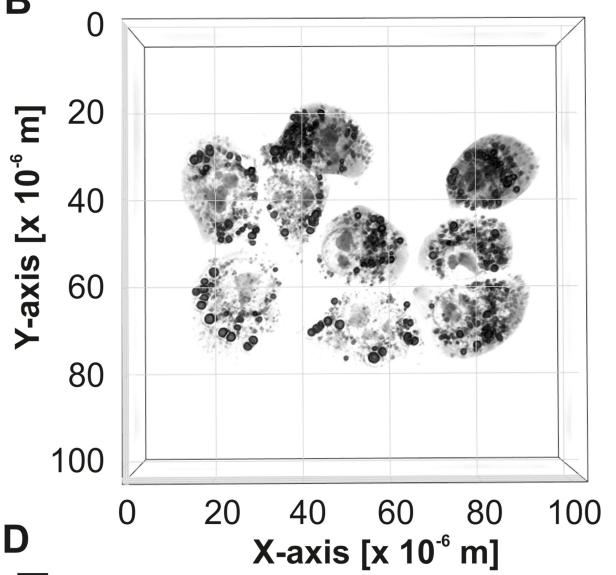

实

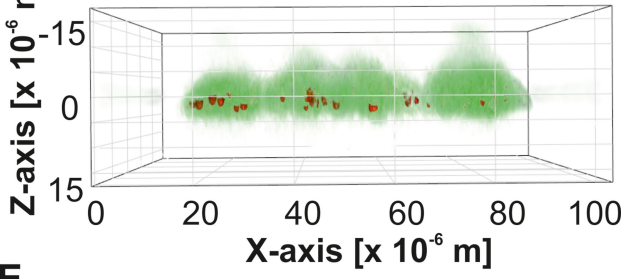

E

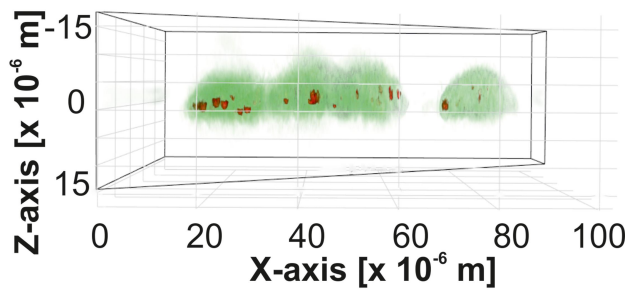

$\mathrm{RI}(\mathrm{I} .2 \mathrm{D}) / \mathrm{RI}(\mathrm{r} .3 \mathrm{D}) \quad \mathrm{SPION}^{\mathrm{LA}-\mathrm{BSA}}(\mathrm{RI}$, dig. $) \quad$ cytoplasm (RI, dig.)

Figure 2 Live-cell-holotomography of cellular nanoparticles. PANC-I SMAD4 $(2-6)^{2}$ cells with unlabeled SPION ${ }^{\text {LA-BSA }}$ are visualized by differences in the refractive index. (A) The 2D image shows an X-Y slice of the RI image. (B-E) The 3D RI images represents the same sample under different angles. (C-E) In the 3D RI images, structures with low RI, such as cytoplasm, cell nuclei and nucleoli, were digitally stained in green, whereas structures with high RI, such as SPION clusters, were digitally stained in red. (b-e) Exemplary RI Images with different viewing angles (B) $90^{\circ}$ (C) $45^{\circ}$ (D) $0^{\circ}$ (E) $0^{\circ}$ and diagonally cut through the Y-axis).

Abbreviations: I., left picture; r., right picture; RI, refractive index; SPION, superparamagnetic iron oxide nanoparticles; SPION ${ }^{\text {LA-BSA }}$, lauric acid- and bovine serum albumin-coated SPIONs.

SPIONs, were plotted against areas of lower RI, which represent cell cytoplasm and nuclei (Figure 3A-E). The threshold values were kept constant for each cell line and defined in such a way that even small amounts of SPIONs are displayed, accepting the risk of false-positive aggregates. Figure $3 \mathrm{~F}$ exhibits the RI-intensity-based SPION amount of the different cell lines, whereas the underlying data are summarized in supplementary Table S1. The discrimination between PaCa DD183 cells incubated with SPIONs and control cells were hardly possible, indicating a very low cellular SPION concentration. The amount of SPIONs in PANC-1 and SUIT-2 cells was still very low, but easier to distinguish from the respective control cells. In contrast, the SPION uptake of PANC-1 ${ }^{\text {SMAD4 (1-4) }}$ and
PANC-1 ${ }^{\text {SMAD4 }}$ (2-6) was significantly higher, indicating a very efficient cellular incorporation of SPIONs.

\section{Cellular SPION Content Determined by MP-AES}

The results of the holotomographic imaging were compared with MP-AES measurements, a reliable and sensitive method to quantify the amount of elementary iron (Figure 4, Table S1). In contrast to techniques based on optical microscopy, MPAES is not dependent of differences in light absorption, contrast, or light scattering. Moreover, atomic spectroscopy is also more suitable than magnetically based methods, such as magnetic particle spectroscopy (MPS), where the quantification of nanoparticles with very low magnetic moments fail. ${ }^{22}$ The 

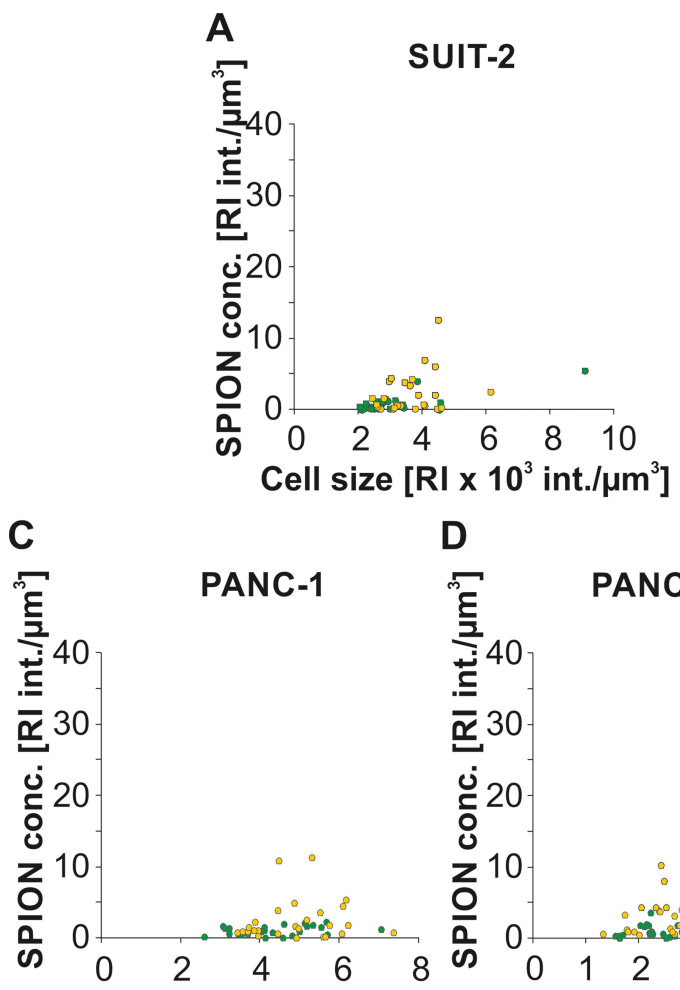

D
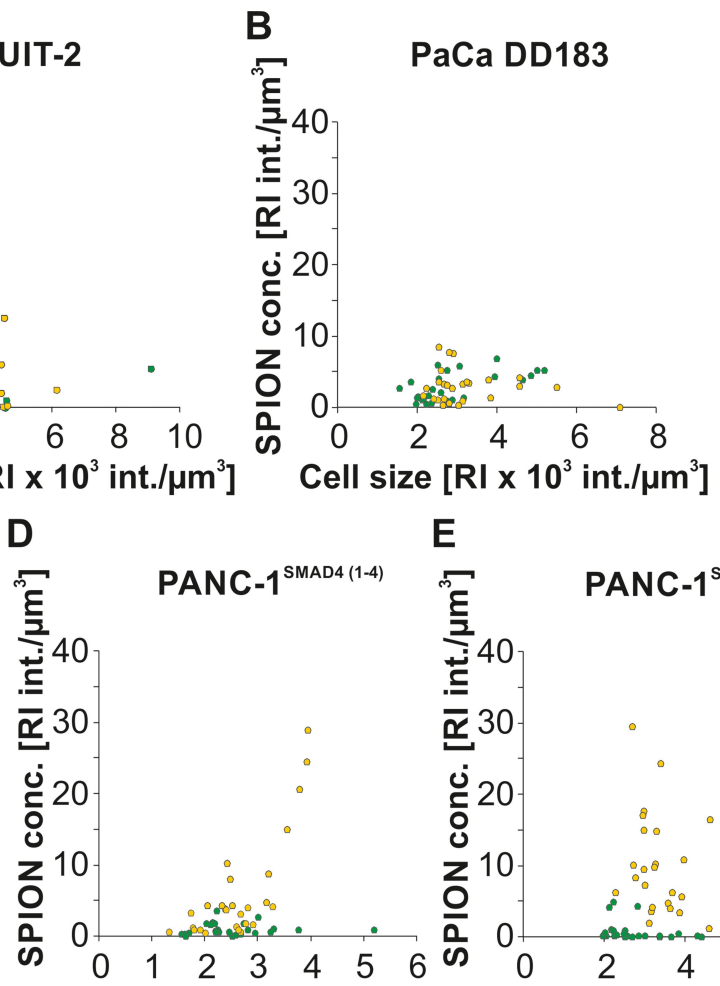

E

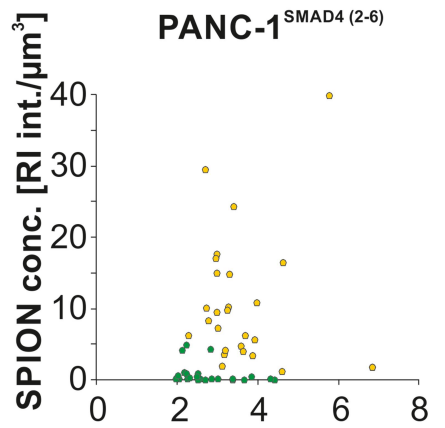

Cell size $\left[R I \times 10^{3} \mathrm{int} . / \mathrm{\mu m}^{3}\right]$ Cell size $\left[R I \times 10^{3} \mathrm{int} . / \mu \mathrm{m}^{3}\right]$ Cell size $\left[R I \times 10^{3} \mathrm{int} . / \mathrm{\mu m}^{3}\right]$

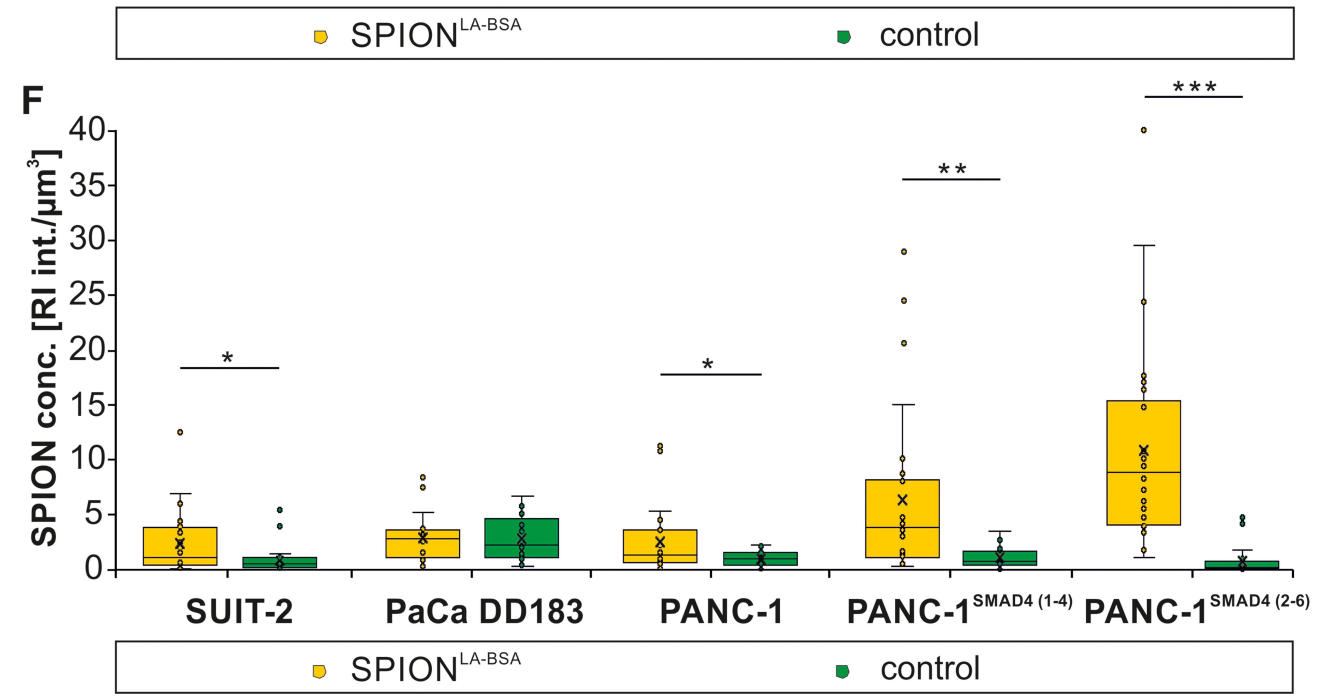

Figure 3 SPION amount determined by holotomographic imaging. SPION accumulations and cell structures were defined as areas of high and low RI, respectively. (A-E) Cell line dependent variations and differences within individual cells in the intensity of areas with high RI. (F) SPION amount as defined by high RI in different pancreatic cell lines treated with and without SPION ${ }^{\text {LA-BSA }}$ particles. Statistical significance are indicated with $*$, $* *$ and $* * *$. The respective confidential intervals are $\mathrm{p}<0.02$, $\mathrm{p}<0.00 \mathrm{I}$ and $\mathrm{P}<0.000 \mathrm{I}$ and were calculated via $t$-test analysis.

Abbreviations: SPION, superparamagnetic iron oxide nanoparticles; SPION ${ }^{\mathrm{LA}-\mathrm{BSA}}$, lauric acid- and bovine serum albumin-coated SPIONs; conc., concentration; RI int., refractive index intensity.

absolute iron amounts in SPION-treated pancreatic cells found by MP-AES were therefore used as a reference to validate the results obtained by optical and holotomographic imaging, as well as the side scatter and viability measured by flow cytometry. As quantified by MP-AES, cellular uptake of SPION $^{\text {LA-BSA }}$ by pancreatic cells corresponded very well with the SPION amount found by holotomographic imaging. However, due to the very sensitive detection limit of MP-AES, the differences between control cells and SPION-treated cells were significantly above the values achieved from the holotomographic image quantification. The data also confirmed the particularly large differences in particle uptake between 


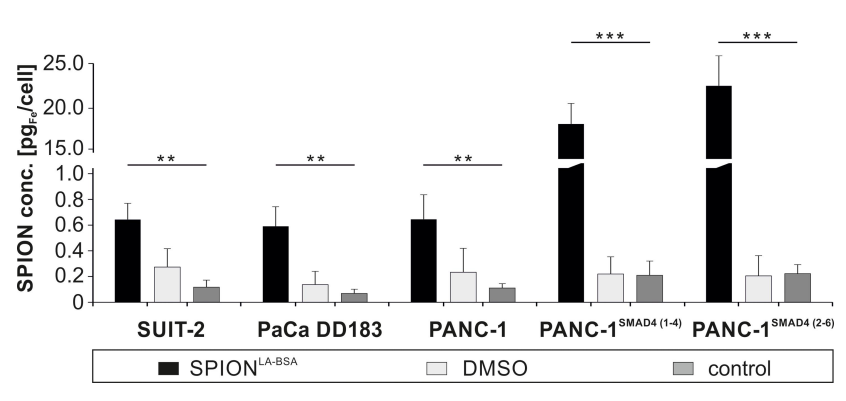

Figure 4 Quantification of cellular nanoparticle load via microwave plasma-atomic emission spectroscopy (MP-AES). Cells were incubated for $24 \mathrm{~h}$ with $100 \mu \mathrm{gg}_{\mathrm{Fe}} / \mathrm{mL}$ SPION ${ }^{\text {LA-BSA }}$ and cell lysates were investigated by MP-AES. The cellular iron concentration ( $\mathrm{Pg}_{\mathrm{Fe}}$ /cell) is shown for SUIT-2, PaCa DDI83, PANC-I, PANC-I SMAD4 (I ${ }^{-4)}$ and PANC-I ${ }^{\text {SMAD4 }}{ }^{(2-6)}$. Control samples contain the corresponding amount of $\mathrm{H}_{2} \mathrm{O}$ or DMSO with a final concentration of $2 \%$ instead of water-based ferrofluid. The data are expressed as the mean \pm standard deviation $(n=4$ with technical quadruplicates). Statistical significance are indicated with $* *$ and $* * *$. The respective confidential intervals are $\mathrm{p}<3 \times 10^{-9}$ and $\mathrm{p}<2 \times 10^{-19}$ and were calculated via $t$-test analysis.

Abbreviations: SPION, superparamagnetic iron oxide nanoparticles; SPION ${ }^{\text {LA- }}$ BSA, lauric acid- and bovine serum albumin-coated SPIONs; conc., concentration; DMSO, dimethyl sulfoxide.

PANC-1 ${ }^{\text {SMAD4 (1-4) }}$ and PANC-1 ${ }^{\text {SMAD4 }}$ (2-6) $^{-1}$ and the other three cell lines.

\section{Cellular SPION Amount Visualized by Fluorescence Microscopy}

Intracellular nanoparticles are often visualized by fluorescent and bright field microscopy. Whereas the localization of fluorescently labelled nanoparticles can easily be detected in cells with fluorescently stained organelles by confocal fluorescence microscopy, the detection of the localization in case of unlabeled particles is still challenging. Optical microscopy only allows plain 2D images, in which the increase of vesicles, imaged by phase contrast or differential interference contrast (DIC), suggest an increase of incorporated particles (Figure 5). Unfortunately, even cells without particle treatment, such as DMSO and control samples, exhibit sites with stronger contrast. This is not only valid for the shown PANC-1 cells (Figure 5), but for all investigated cells, although some cell lines show fewer areas with high contrast in SPION-untreated samples (Figures S3-S6). Consequently, with optical microscopy, the uptake of unlabeled particles can only be concluded on images showing cells with high particle amounts. Interestingly, the findings are in agreement with the results from the holotomographic imaging. With both imaging methods, obvious differences between particle-treated and untreated cells could not be found in PaCa DD183 cells, whereas differences were clearly present within PANC-1 and SUIT-2 cells. These were even more prominent in
PANC-1 ${ }^{\text {SMAD4 }(1-4)}$ and PANC-1 ${ }^{\text {SMAD4 (2-6) }}$ cells were the vesicle amount was highly accumulated (Figures S5 and

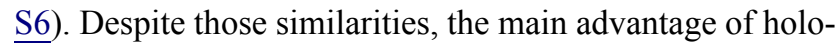
tomographic imaging is the $3 \mathrm{D}$ illustration and the possibility to determine whether the particle are indeed accumulated within the cell body.

\section{Cellular SPION Load Determined by Flow Cytometry}

Flow cytometry is commonly used to determine and quantify biochemical and physical properties of cells. Due to the characteristic light scattering of cells and their components, cells can be morphologically characterized by forward scatter (FSc) and side scatter (SSc). Whereas the FSc delivers data corresponding to the cell size, the SSc is related to the granularity of cells, which is increased by cellular nanoparticles. However, the direct correlation between SSc and particle amount is only valid in viable cells, as the granularity strongly changes during cell death. Thus, gating on healthy cells, characterized either by negative annexin $\mathrm{V}$ and negative propidium iodide staining, or positive DiIC1(5) and positive Hoechst staining, the correlation between SSc increase and particle amount can be used for the determination of the cellular particle quantity by flow cytometry (Figure S1). ${ }^{22}$

To investigate the uptake of cellular particles in different pancreatic cell lines, the cells were treated with SPION $^{\text {LA-BSA }}$ for $24 \mathrm{~h}$. Following sample preparation, the side scatter intensity of the cell populations was determined by flow cytometry (Figure 6). The average SSc intensity of cell populations treated with particles was significantly higher than untreated cells, or cells treated with DMSO, resulting in a right shift of the SSc curves (Figure 6A-E). This shift was less pronounced with PaCa DD183 and SUIT-2 cells than with PANC-1, PANC-1 ${ }^{\text {SMAD4 }}$ (1-4) $^{2}$ and PANC-1 ${ }^{\text {SMAD4 }}$ (2-6), indicating major morphological changes in PANC-1 cells and derivatives upon SPION treatment compared to the other investigated cell lines. Moreover, SSc measurement revealed only negligible differences between control cells and DMSO-treated cells (Figure 6A-F). Thus, an increase of the arithmetic SSc mean after the particle treatment is a strong indicator of the presence of cellular particles and not a result of toxic effects. Compared to MP-AES, which allows an exact quantification of the SPION-based iron amount, SSc measurements mainly allow an evaluation of the particle uptake (Figure 6F, Table S1). However, a quantitative determination of the cellular particle amount by flow cytometry is possible once a specific cell type 


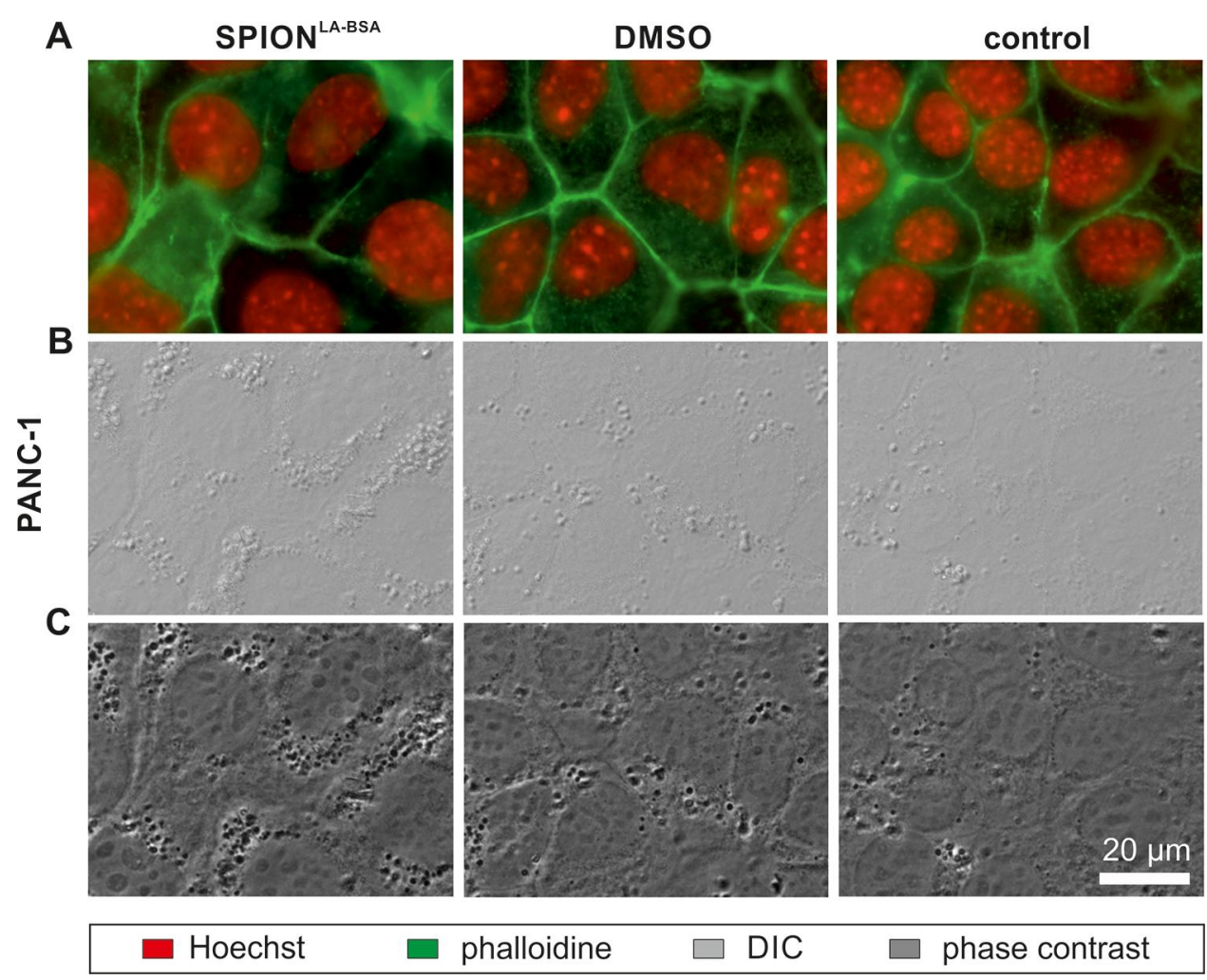

Figure 5 SPION load visualized by optical imaging. PANC-I cells were treated with unlabeled SPION ${ }^{\text {LA-BSA }}\left(100 \mu g_{\mathrm{Fe}} / \mathrm{mL}\right)$ or with the corresponding amount of $\mathrm{H}_{2} \mathrm{O}$ or DMSO (final concentration of $2 \%$ ) for $24 \mathrm{~h}$ and visualized by (A) fluorescent staining for nuclei (Hoechst 33342, red) and actin cytoskeleton (Alexa Fluor 488 Phalloidin, green), (B) differential interference contrast (DIC) and (C) phase contrast. Compared to few spots with high contrast within control and DMSO samples, numerous areas of high contrast indicate SPION accumulations within vesicles in nanoparticle-treated cells.

Abbreviations: SPION, superparamagnetic iron oxide nanoparticles; SPION ${ }^{\mathrm{LA}-\mathrm{BSA}}$, lauric acid- and bovine serum albumin-coated SPIONs; DMSO, dimethyl sulfoxide; DIC, differential interference contrast.

and the concentration of a defined particle system have been normalized against each other. ${ }^{10}$

\section{Discussion}

Apart from the intrinsic properties of the involved cells, the physicochemical properties of nanoparticles are decisive for the cellular uptake and toxicity of the particles. Different medical applications can therefore place very different demands on the particle properties, such as extended blood circulation time. This becomes even more complicated, if imaging is intended to detect or exclude organs that lie behind cellular barriers, such as the blood-brain barrier or the placenta. On the other hand, particles intended for cancer treatment and coupled with chemotherapeutic agents can fulfil their task more efficiently if they are incorporated into the cell and not only associates with the cell membrane. However, the uptake is strongly dependent on the physicochemical properties of the particles, which can be significantly altered by attached substances. These properties are not only affected by the formation of a protein corona, which usually occurs in the presence of physiological fluids, such as blood. They are especially dependent on the original particle composition, such as stabilization by polymers or proteins, functionalization with therapeutics, as well as by the binding of fluorescent markers. Thus, predicting the behavior of unlabelled particles, including cellular binding, uptake and accumulation, by using their fluorescently labelled counterparts is a quite unreliable and unsuitable approach. Consequently, there is an urgent need for a reliable visualization of unlabelled particles. In particular, an accurate determination of the SPION localization and SPION quantity is required to clarify and understand particle-based effects on cells. Still, the majority of laboratories do not have access to the equipment that allow the $3 \mathrm{D}$ localization of label-free particles. A possible method for the visualization of label-free particles is holotomographic microscopy. The underlying refractive index-based technique allows the 3D localization of structures with different refractive indexes, eg, nanoparticle-filled vesicles. However, the sole definition of existing particle agglomerates by a high refractive index is not absolutely accurate, 

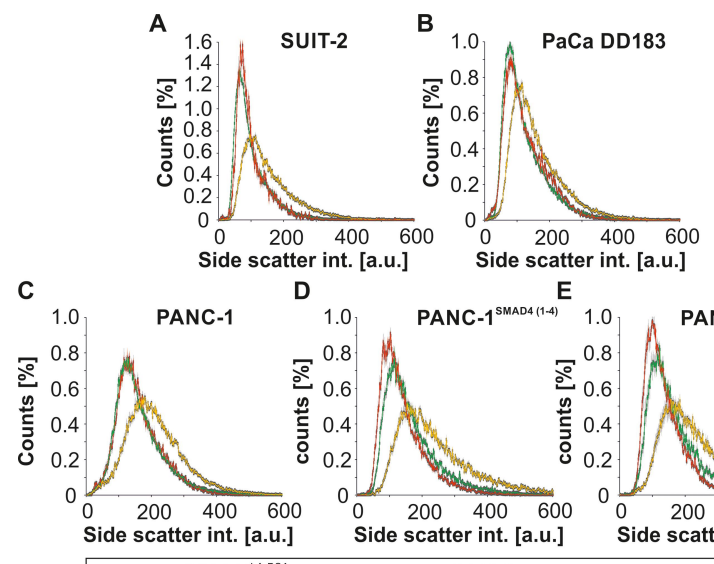

E

E 1.0
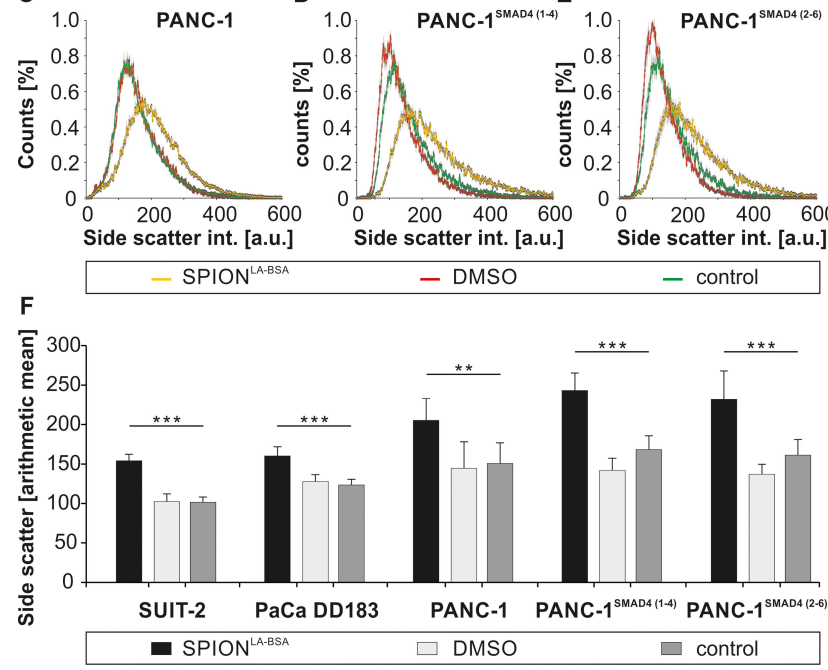

Figure 6 Cellular side scatter increase after SPION treatment measured by flow cytometry. (A-F) Pancreatic cell lines were treated with SPION ${ }^{\text {LA-BSA }}\left(100 \mu g_{\mathrm{Fe}}\right.$ $/ \mathrm{mL}$ ) for $24 \mathrm{~h}$ and analyzed by flow cytometry. Control samples contained the respective amount of ultrapure water and the toxicity control $2 \%$ DMSO. (A-E) Distribution of the cellular SSC intensity. The total cell numbers of each sample were set to $100 \%$. Grey error bars indicate the standard error of $n=4$ with quadruplicates. (F) The arithmetic mean of the SSc intensity in different pancreatic cell lines. Error bars indicate the standard deviation of $n=4$ with quadruplicates. Statistical significance are indicated with $* *$ and $* * *$. The respective confidential intervals are $\mathrm{p}<2 \times 10^{-4}$ and $\mathrm{p}<2 \times 10^{-7}$ and were calculated via $t$-test analysis. Abbreviations: SPION, superparamagnetic iron oxide nanoparticles; SPION ${ }^{\text {LA-BSA }}$, lauric acid- and bovine serum albumin-coated SPIONs; DMSO, dimethyl sulfoxide; int., intensity; a.u., arbitrary unit.

as there are cellular structures that have an increased refractive index even though the cells have not been treated with nanoparticles. A possible solution is the setting of a limit for the intensity of the refractive index which is high enough that all false-positive cells are no longer considered. However, this would mean that cells with smaller particle aggregates would no longer be included. We have therefore set this exclusion limit as low as necessary, even with the risk of detecting fewer false-positive particle aggregates. Even so, a direct comparison of SPION-treated and untreated cells allows a valid statement about particle uptake. However, the reliability of RI-based determination of the particle amount is higher, the higher the RI of the nanoparticles are, and the more particles are bound to or incorporated into cells. Hence, the general translatability of this technique to other nanoparticles is given, if the RI of the particle aggregates differs significantly from the RI of other cell compartments. The method is mainly applicable for the representation of particle aggregates in flat, adherent cells. Cells growing in several cell layers or spherical clusters of cells are less suitable, since the RI is strongly influenced by the increasing cell thickness and cannot be reliably distinguished from the existing particle-filled vesicles.

The visualization of label-free particles by optical microscopy exhibit similar problems (Figures 5 and $\underline{\mathrm{S}}-$

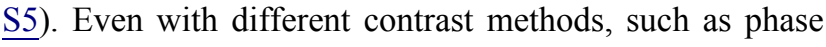
contrast and DIC, an exact differentiation between particle-filled and normal vesicles is not possible. Only an increased number of vesicles makes particle uptake appear likely. The great advantage of holotomographic microscopy over optical microscopy, however, is the 3D representation of particle aggregates and the ability to distinguish between membrane-bound and incorporated particles.

To further validate the results from holotomographic imaging and analysis, we utilized a parallel investigation by flow cytometry, which is a widely used method to investigate the influence of particles or toxic substances on cells. ${ }^{25,31}$ Meanwhile, there are already several reports about a correlation between increasing SSc and the cellular amount of metal and metal oxides..$^{10,22,32-35}$ However, even this method is not able to differentiate between intracellular and membrane-bound particle aggregates. Nevertheless, flow cytometry is indispensable for a thorough characterization of particles' impact upon cells, especially as it allows the analysis of many different toxicity markers simultaneously.

Interestingly, this work also revealed that the investigated pancreatic cells responded very differently to the particle treatment. Although the cytotoxicity of SPION ${ }^{\mathrm{LA}-}$ ${ }^{\mathrm{BSA}}$ was generally very low, the cytotoxicity was slightly increased in SUIT-2 cells, while the effect of SPIONs on PANC-1 ${ }^{\text {SMAD4 (1-4) }}$ was minimal, or undetectable in other cell lines. This shows that the toxicity is not only dependent on the particle type but also to a large extent on the cellular sensitivity of the involved cells. The toxicity studies show how complex and unpredictable the cellular effect of particle treatment can be and, consequently, how important it is to study the potential toxicity of particles. As a consequence, preclinical studies should include extensive experiments not only with one cell type but with several different cell types, because the future clinical applications also involve different tissues and cells. 
Although all methods, including holotomographic imaging, optical microscopy, flow cytometry and atomic emission spectroscopy, measured similar differences in cellular particle uptake, only the holotomographic technique allows the determination of particle localization in 3D. Although the cells are morphologically very similar and have an epitheliallike character, the uptake efficiency of PaCa DD183, SUIT-2 and PANC-1 was relatively low, while PANC-1 1 SMAD4 (1-4) and PANC-1 ${ }^{\text {SMAD4 (2-6) }}$ show an extremely increased particle internalization. This finding is all the more interesting, as PANC-1 ${ }^{\text {SMAD4 (1-4) }}$ and PANC-1 ${ }^{\text {SMAD4 (2-6) }}$ differ from the PANC-1 cell line only by a mutation in the SMAD4 gene. It is known that albumin can emulate TGF-b signaling, ${ }^{36}$ but the connection between SMAD4 negative cells and increased uptake is rather new and interesting.

\section{Conclusion}

In conclusion, we demonstrated that holotomographic imaging is a valid method to determine the cellular amount of label-free SPIONs and to specify the 3D localization of particle aggregates. Intracellular SPION amounts detected with the holotomographic technique closely resemble the iron amounts measured by the highly precise MP-AES technique. Both methods deliver comparable results for the cellular SPION content in different pancreatic cell lines. Although MP-AES is much more accurate in the quantification of the actual iron amount, only the holotomographic imaging has the capacity to make statements about the cellular localization of particles.

\section{Acknowledgments}

We thank Ralf Meister, Munich, Germany for kindly donating the holotomographic microscope system. This study was supported by the Deutsche Forschungsgemeinschaft and the Forschungsstiftung Medizin, University Hospital Erlangen, Germany. J. Band and A. Kerpes are gratefully acknowledged for their help and technical expertise in the cell culture. Dr. I. Cicha is gratefully acknowledged for help with English editing.

\section{Disclosure}

The authors report no conflicts of interest in this work.

\section{References}

1. Jin R, Lin B, Li D, Ai H. Superparamagnetic iron oxide nanoparticles for MR imaging and therapy: design considerations and clinical applications. Curr Opin Pharmacol. 2014;18:18-27. doi:10.1016/j. coph.2014.08.002
2. Thomas R, Park IK, Jeong YY. Magnetic iron oxide nanoparticles for multimodal imaging and therapy of cancer. Int J Mol Sci. 2013;14 (8):15910-15930. doi:10.3390/ijms140815910

3. Tietze R, Zaloga J, Unterweger H, et al. Magnetic nanoparticle-based drug delivery for cancer therapy. Biochem Biophys Res Commun. 2015;468(3):463-470. doi:10.1016/j.bbrc.2015.08.022

4. Blanco-Andujar C, Walter A, Cotin G, et al. Design of iron oxide-based nanoparticles for MRI and magnetic hyperthermia. Nanomedicine (Lond). 2016;11(14):1889-1910. doi:10.2217/nnm-2016-5001

5. Rad AM, Janic B, Iskander AS, Soltanian-Zadeh H, Arbab AS. Measurement of quantity of iron in magnetically labeled cells: comparison among different UV/VIS spectrometric methods. Biotechniques. 2007;43(5):627-628, 630, 632 passim. doi:10.2144/000112599

6. Wiekhorst F, Steinhoff U, Eberbeck D, Trahms L. Magnetorelaxometry assisting biomedical applications of magnetic nanoparticles. Pharm Res. 2012;29(5):1189-1202. doi:10.1007/s11095-011-0630-3

7. Tietze R, Jurgons R, Lyer S, et al. Quantification of drug-loaded magnetic nanoparticles in rabbit liver and tumor after in vivo administration. $J$ Magn Magn Mater. 2009;321:1465-1468. doi:10.1016/j.jmmm.2009.02.068

8. Canfranc E, Abarca A, Sierra I, Marina ML. Determination of iron and molybdenum in a dietetic preparation by flame AAS after dry ashing. J Pharm Biomed Anal. 2001;25(1):103-108. doi:10.1016/ S0731-7085(00)00487-8

9. Dadashzadeh ER, Hobson M, Henry Bryant L, Dean DD, Frank JA Rapid spectrophotometric technique for quantifying iron in cells labeled with superparamagnetic iron oxide nanoparticles: potential translation to the clinic. Contrast Media Mol Imaging. 2013;8 (1):50-56. doi:10.1002/cmmi.1493

10. Poller JM, Zaloga J, Schreiber E, et al. Selection of potential iron oxide nanoparticles for breast cancer treatment based on in vitro cytotoxicity and cellular uptake. Int $J$ Nanomedicine. 2017;12:3207-3220. doi:10.2147/IJN.S132369

11. Gunn J, Paranji RK, Zhang M. A simple and highly sensitive method for magnetic nanoparticle quantitation using $1 \mathrm{H}-\mathrm{NMR}$ spectroscopy. Biophys J. 2009;97(9):2640-2647. doi:10.1016/j.bpj.2009.08.013

12. Mayhew TM, Muhlfeld C, Vanhecke D, Ochs M. A review of recent methods for efficiently quantifying immunogold and other nanoparticles using TEM sections through cells, tissues and organs. Ann Anat. 2009;191(2):153-170. doi:10.1016/j.aanat.2008.11.001

13. Burrows ND, Penn RL. Cryogenic transmission electron microscopy: aqueous suspensions of nanoscale objects. Microsc Microanal. 2013;19(6):1542-1553. doi:10.1017/S1431927613013354

14. Stender AS, Marchuk K, Liu C, et al. Single cell optical imaging and spectroscopy. Chem Rev. 2013;113(4):2469-2527. doi:10.1021/ cr300336e

15. Yin L, Wang W, Wang S, Zhang F, Zhang S, Tao N. How does fluorescent labeling affect the binding kinetics of proteins with intact cells? Biosens Bioelectron. 2015;66:412-416. doi:10.1016/j. bios.2014.11.036

16. Peterson AW, Halter M, Tona A, Plant AL. High resolution surface plasmon resonance imaging for single cells. BMC Cell Biol. 2014;15:35. doi:10.1186/1471-2121-15-35

17. Spicer GLC, Almassalha L, Martinez IA, et al. Label free localization of nanoparticles in live cancer cells using spectroscopic microscopy. Nanoscale. 2018;10(40):19125-19130. doi:10.1039/C8NR07481J

18. Patskovsky S, Bergeron E, Meunier M. Hyperspectral darkfield microscopy of PEGylated gold nanoparticles targeting CD44-expressing cancer cells. J Biophotonics. 2015;8(1-2):162-167. doi:10.1002/jbio.201300165

19. Patskovsky S, Bergeron E, Rioux D, Meunier M. Wide-field hyperspectral 3D imaging of functionalized gold nanoparticles targeting cancer cells by reflected light microscopy. J Biophotonics. 2015;8 (5):401-407. doi:10.1002/jbio.201400025

20. Cotte Y, Toy F, Jourdain P, et al. Marker-free phase nanoscopy. Nat Photonics. 2013;7(2):113-117. doi:10.1038/nphoton.2012.329 
21. Zaloga J, Janko C, Nowak J, et al. Development of a lauric acid/ albumin hybrid iron oxide nanoparticle system with improved biocompatibility. Int $J$ Nanomedicine. 2014;9:4847-4866. doi:10.2147/IJN.S68539

22. Friedrich RP, Janko C, Poettler M, et al. Flow cytometry for intracellular SPION quantification: specificity and sensitivity in comparison with spectroscopic methods. Int $J$ Nanomedicine. 2015;10:4185-4201. doi:10.2147/IJN.S82714

23. Ran FA, Hsu PD, Wright J, Agarwala V, Scott DA, Zhang F. Genome engineering using the CRISPR-Cas9 system. Nat Protoc. 2013;8 (11):2281-2308. doi:10.1038/nprot.2013.143

24. Ruckert F, Aust D, Bohme I, et al. Five primary human pancreatic adenocarcinoma cell lines established by the outgrowth method. J Surg Res. 2012;172(1):29-39. doi:10.1016/j.jss.2011.04.021

25. Munoz LE, Maueroder C, Chaurio R, Berens C, Herrmann M, Janko C. Colourful death: six-parameter classification of cell death by flow cytometry-dead cells tell tales. Autoimmunity. 2013;46 (5):336-341. doi:10.3109/08916934.2012.755960

26. Zaloga J, Stapf M, Nowak J, et al. Tangential flow ultrafiltration allows purification and concentration of lauric acid-/albumin-coated particles for improved magnetic treatment. Int J Mol Sci. 2015;16 (8):19291-19307. doi:10.3390/ijms160819291

27. van Engeland M, Nieland LJ, Ramaekers FC, Schutte B, Reutelingsperger CP. Annexin V-affinity assay: a review on an apoptosis detection system based on phosphatidylserine exposure. Cytometry. 1998;31(1):1-9. doi:10.1002/(SICI)1097-0320(19980101)31:1<1::AID-CYTO1>3.0.CO;2-R

28. Demchenko AP. The change of cellular membranes on apoptosis: fluorescence detection. Exp Oncol. 2012;34(3):263-268.

29. Bohm I, Traber F, Block W, Schild H. [Molecular imaging of apoptosis and necrosis - basic principles of cell biology and use in oncology]. Rofo. 2006;178(3):263-271.
30. Lugert S, Unterweger H, Muhlberger M, et al. Cellular effects of paclitaxel-loaded iron oxide nanoparticles on breast cancer using different $2 \mathrm{D}$ and $3 \mathrm{D}$ cell culture models. Int $J$ Nanomedicine. 2019;14:161-180. doi:10.2147/IJN.S187886

31. Janko C, Munoz L, Chaurio R, et al. Navigation to the graveyard-induction of various pathways of necrosis and their classification by flow cytometry. Methods Mol Biol. 2013;1004:3-15.

32. Xia J, Zhang S, Zhang Y, et al. The relationship between internalization of magnetic nanoparticles and changes of cellular optical scatter signal. J Nanosci Nanotechnol. 2008;8(12):6310-6315. doi:10.1166/ jnn.2008.18387

33. Suzuki H, Toyooka T, Ibuki Y. Simple and easy method to evaluate uptake potential of nanoparticles in mammalian cells using a flow cytometric light scatter analysis. Environ Sci Technol. 2007;41 (8):3018-3024. doi:10.1021/es0625632

34. Zucker RM, Daniel KM, Massaro EJ, Karafas SJ, Degn LL, Boyes WK. Detection of silver nanoparticles in cells by flow cytometry using light scatter and far-red fluorescence. Cytometry A. 2013. doi:10.1002/cyto.a.22342

35. Toduka Y, Toyooka T, Ibuki Y. Flow cytometric evaluation of nanoparticles using side-scattered light and reactive oxygen species-mediated fluorescence-correlation with genotoxicity. Environ Sci Technol. 2012;46(14):7629-7636. doi:10.1021/ es300433x

36. Siddiqui SS, Siddiqui ZK, Malik AB. Albumin endocytosis in endothelial cells induces TGF-beta receptor II signaling. Am $J$ Physiol Lung Cell Mol Physiol. 2004;286(5):L1016-1026. doi:10.1152/ajplung.00356.2003

\section{Publish your work in this journal}

Nanotechnology, Science and Applications is an international, peerreviewed, open access journal that focuses on the science of nanotechnology in a wide range of industrial and academic applications. It is characterized by the rapid reporting across all sectors, including engineering, optics, bio-medicine, cosmetics, textiles, resource sustainability and science. Applied research into nano-materials, particles, nano-structures and fabrication, diagnostics and analytics, drug delivery and toxicology constitute the primary direction of the journal. The manuscript management system is completely online and includes a very quick and fair peer-review system, which is all easy to use. Visit http://www.dovepress.com/testimonials.php to read real quotes from published authors. 